\title{
Farmacocinética e mecanismos de teratogenicidade dos medicamentos na gestação: uma revisão da literatura
}

\author{
Pharmacokinetics and teratogenicity mechanisms \\ of medicines in pregnancy: a review
}

Recebido em: 17/10/2016

Aceito em: $\quad 31 / 03 / 2017$
Andréia Moreira ANDRADE ${ }^{1}$; Alanderson Alves RAMALHO ${ }^{1}$; Simone Perufo

OPITZ $^{1}$; Fernanda Andrade MARTINS ${ }^{1}$; Rosalina Jorge KOIFMAN ${ }^{2}$

${ }^{1}$ Universidade Federal do Acre. BR 364, KM 4,5 Distrito Industrial, CEP 69.920-900.

Rio Branco, Acre, Brasil. 'Escola Nacional de Saúde Pública, FIOCRUZ, R. Leopoldo

Bulhões, 14808 Andar, - Bonsucesso, CEP 21041-210. Rio de Janeiro, RJ, Brasil.

E-mail:amasmsbg@hotmail.com

\section{ABSTRACT}

The presence of emerging contaminants in surface Physiological changes that occur during a gestational period on a female organism are greatly explored by researchers and it is well elucidated by scientific literature, however, the theme: pharmacokinetics and mechanisms of teratogenicity by medicines on female organisms passing by physiological changes, it is still poorly explored. The research's purpose was to review and contribute to adding scientific knowledge about pharmacokinetics and mechanisms of teratogenicity by drugs used during gestation.

KEYWORDS: Pharmacokinetics; Abnormalities, Drug-Induced; Pregnancy.

\section{RESUMO}

As alterações fisiológicas que ocorrem no organismo feminino durante o período gestacional é assunto bastante explorado pelos pesquisadores e encontra-se bem elucidado pela literatura; porém a temática relativa à farmacocinética e os mecanismos de teratogenicidade dos fármacos neste organismo em processo de alterações fisiológicas ainda encontra-se pouco explorado. O objetivo desta pesquisa foi revisar e contribuir para ampliar o conhecimento científico acerca da farmacocinética e dos mecanismos de teratogenicidade dos fármacos na gestação.

PALAVRAS-CHAVE: Farmacocinética; Anormalidades Induzidas por Medicamentos; Gravidez.

\section{INTRODUÇÃO}

O pré-natal constitui o primeiro contato da gestante e do concepto com os serviços de saúde. Esse acompanhamento visa manter a integridade da saúde de ambos e é crucial para estabelecer medidas de intervenção em casos em que há detecção precoce de morbidades e de situações de exposição nociva a agentes teratogênicos. Além disso, a realização de um pré-natal adequado, com número de consultas suficientes, com a realização dos exames pertinentes, oferecendo os medicamentos essen- ciais necessários, é uma forma de garantir uma gestação saudável e um parto seguro $(1,2)$.

Sendo assim, para dispensar um atendimento adequado às necessidades da gestante e do recém-nascido, é necessário que os profissionais de saúde detenham o conhecimento básico sobre a classificação de risco em que determinados medicamentos se enquadram. Esses riscos podem ser avaliados considerando o período gestacional de maior risco para o consumo de medicamentos; se os metabólitos destes medicamentos são capazes de atravessar a barreira placentária ou serem encontrados no 
leite materno; e se é realmente necessário dispensar um tratamento medicamentoso (3).

Diante disso, descrever o comportamento dos medicamentos no organismo materno-infantil, durante a gestação, e desenvolver estudos epidemiológicos abordando o uso destes medicamentos são estratégias que contribuem com a consolidação do conhecimento e oferecem subsídios para que os profissionais possam tomar decisões pautadas em documentos técnicos (4).

A dificuldade ética de se realizar ensaios clínicos com gestantes e as dúvidas quanto aos riscos para o concepto são argumentos que contrastam com a necessidade de medicar mulheres grávidas. Nesse sentido, se destaca a importância de estudos de revisão de literatura, bem como abordagens epidemiológicas, sobre a utilização de medicamentos em gestantes que possam subsidiar a prescrição dos mesmos (5). Assim, o objetivo deste estudo foi revisar e contribuir para ampliar o conhecimento científico acerca da farmacocinética e dos mecanismos de teratogenicidade dos medicamentos na gestação. Para isto, foi utilizada a técnica de revisão da literatura nas bases de periódicos nacionais e internacionais da Capes.

Farmacocinética dos medicamentos. A Farmacocinética é a área da Farmacologia que descreve os mecanismos de passagem dos medicamentos pelo organismo, não se prendendo aos sítios de ação, mas às etapas que se intercalam desde a absorção até a sua excreção (4).

O conhecimento das alterações fisiológicas no período gestacional e sua influência na farmacocinética dos medicamentos é indispensável para promoção de uma farmacoterapia efetiva e segura (6). As substâncias farmacológicas são capazes de atravessar a placenta e atingir o feto, algumas em maior grau do que outras. No entanto, esse processo depende de alguns fatores como: propriedades físico-químicas do fármaco; velocidade e quantidade de ativo que atravessa a placenta; duração da exposição ao fármaco; características de distribuição nos tecidos fetais, estágio do desenvolvimento gestacional, sinergismo ou antagonismo com outros fármacos simultaneamente administrados, constituição genética, suscetibilidade particular do feto, estado de nutrição e saúde da mãe (7-9).

A placenta, assim como as demais membranas do organismo, possui uma composição que permite uma seletividade que depende da característica de lipossolubilidade dos medicamentos, ou seja, substâncias lipofílicas tendem a sofrer difusão rápida na placenta e penetrar na circulação fetal (10). Esta seria uma propriedade carac- terística do tiopental, um anestésico da classe dos barbitúricos, utilizado em casos de cesarianas, o qual é capaz de atravessar a placenta rapidamente e levar o recém-nascido a estados de sedação ou apneia (11).

A velocidade de transferência e a quantidade de medicamento que atravessam a placenta sofrem influência do seu peso molecular. Substâncias com peso molecular de 250-500 Da atravessam sem dificuldades a placenta, enquanto as substâncias com peso acima de $1.000 \mathrm{Da}$ não conseguem atravessá-la. Com base nesse conhecimento, a heparina é o anticoagulante de escolha para utilização em possíveis complicações durante a gestação, pois o seu alto peso molecular e sua polaridade, dificultam sua passagem pela barreira placentária, oferecendo maior segurança ao concepto $(3,12)$.

Outro meio de contribuir para segurança do concepto é a existência de transportadores placentários, cuja descoberta permitiu elucidar mecanismos de proteção do feto contra a ação de medicamentos. Como exemplo, tem-se a ação sobre a digoxina, que é bombeada de volta à circulação materna por meio de uma proteína denominada P-glicoproteína (13).

O conhecimento de algumas alterações fisiológicas maternas que ocorrem durante a gestação e que podem influenciar na farmacocinética dos medicamentos é importante (13).

Durante a gestação, por mecanismos ainda não completamente elucidados, o sistema cardiovascular apresenta um aumento de 40 a $50 \%$ do volume sanguíneo, aumento do volume hídrico corporal total, aumento do débito cardíaco e, consequentemente, aumento do volume de distribuição de medicamentos hidrofílicos. Essa alteração acarreta uma diminuição da concentração circulante desse tipo de medicamento, necessitando assim de incremento na dose inicial e de manutenção de fármacos hidrofílicos para obter concentração plasmática terapêutica $(9,12,14)$.

A capacidade de se ligarem às proteínas plasmáticas maternas, principalmente à albumina, é uma característica que faz com que fármacos como as sulfonamidas, a fenitoína, o midazolan e os anestésicos locais, que possuem alta afinidade por esta proteína, não estejam disponíveis para transferência ao feto, constituindo, assim, uma alternativa para prescrição médica durante a gestação. Porém, com o aumento do volume sanguíneo, pode ocorrer uma diminuição na concentração das proteínas plasmáticas, principalmente a albumina, nesse caso, como diminui a quantidade de proteínas livres, os fármacos que possuem alta afinidade de ligação ficarão 
mais biodisponíveis e haverá aumento em sua concentração circulante. Contudo, esse fato não inviabiliza sua utilização por gestantes, uma vez que a meia-vida de eliminação dos metabólitos é menor que uma hora $(8,12,14)$.

O aumento do volume sanguíneo e a vasodilatação dos canais aferentes e eferentes dos néfrons elavam a taxa de filtração glomerular em torno de 50\%. Este fator, é responsável pelo aumento do fluxo sanguíneo que acelera o processo de início da absorção e ação de fármacos administrados via intramuscular (13). Este mecanismo também acarreta uma eliminação mais rápida e eficiente dos metabólitos, resultando em meias-vidas mais curtas e em alguns casos levando a dosagens subterapêuticas. Alguns fármacos fazem parte deste grupo tais como: lítio, ampicilina, cefuroxima, cefazolina, piperacilina, atenolol, digoxina e outros $(6,12,13)$.

A gestação promove ganho de peso e em alguns casos excesso de gordura corporal. Neste sentido, a utilização de fármacos com maior coeficiente de lipossolubilidade aumenta o seu processo de distribuição, sendo importante avaliar a utilização de fármacos como a succinilcolina, que tem lipossolubilidade aumentada e geralmente é utilizada em cesarianas (14).

$\mathrm{O}$ aumento nos níveis de estrogênio circulante promove alterações anatômicas no sistema respiratório, aumentando a vascularização e o edema nas mucosas. Embora a toxicidade não seja comprovada experimentalmente, sabe-se que há aumento de absorção de fármacos inalatórios por mulheres gestantes $(11,12)$.

O tempo de esvaziamento gástrico na gestação é reduzido devido à ação da progesterona. Essa demora, somada à compressão do estômago pelo útero gravídico, promove aumento da pressão gástrica e esta alteração influencia o processo de absorção dos fármacos de uso oral, fazendo com que o início do efeito desejado seja retardado. Estas situações devem ser observadas em especial quando se deseja atingir a concentração máxima terapêutica de um fármaco em menor tempo (12-14).

A farmacocinética foi descrita por diversos parâmetros relacionados tanto às características do fármaco quanto às alterações fisiológicas no organismo durante a gestação. Todos esses fatores devem ser observados no momento da decisão da prescrição de um fármaco, pela importância de uma avaliação criteriosa quanto ao risco/ benefício. A reação do concepto às substâncias farmacológicas se apresenta de forma diferente, uma vez que, para eles, essas substâncias podem levar a elevada toxicidade devido à permeabilidade aumentada da barreira hematoencefálica, bem como a deficiente conjugação enzimática no fígado (15).

Teratogenicidade dos medicamentos. Farmacoteratologia é um ramo da farmacologia destinado ao estudo de anomalias congênitas produzidas por uso de medicamentos durante o ciclo gestacional. Após a descoberta dos medicamentos teratogênicos clássicos, tais como a talidomida e o dietilestilbestrol, e até mesmo os casos de anomalias causados pelo vírus da rubéola, o uso de medicamentos durante o período gestacional tornou-se mais criterioso (16-19).

Teratógenos são agentes que alteram de forma irreversível o desenvolvimento estrutural ou funcional do embrião ou feto, e podem ser de diversas naturezas ou origens (20-22). Algumas características são comuns aos teratógenos como: provocar má formações, indicando seletividade para determinado órgãos-alvo; exercer seus efeitos em determinado estágio do desenvolvimento embrionário ou fetal; apresentar uma incidência de teratogenicidade dose-dependente ou ser comprovado como agente teratógeno por meio de um modelo experimental animal (23).

A análise de um teratógeno deve ser pautada em diversos aspectos e essa é uma questão que tem sido debatida e corrobora com a limitação da classificação de risco estabelecida pelo FDA, que abrange apenas a análise dos riscos ligada ao medicamento $(7,19,20)$.

Devem ser analisadas as seguintes características para se estabelecer um teratógeno humano: 1) científicas, em que as evidências de malformação devem ser comprovadas por dois ou mais estudos epidemiológicos de elevada qualidade; 2) análise comprovada de efeito dose-resposta ligada ao agente em questão; 3 ) efeito limiar, ou seja, todos os agentes possuem uma dose mínima e que pode ser considerada segura para exposição ao feto; 4) o período gestacional, sendo que o primeiro trimestre é considerado o de maior vulnerabilidade, porém não é regra geral, uma vez que existem agentes que atuam especificamente em outros períodos da gestação; 5) a via de contato do agente com o organismo da gestante, uma vez que, existem medicamentos considerados seguros para uso dérmico e totalmente contraindicados para uso oral; 6) a forma de estabelecimento da dose teratogênica em humanos, que geralmente é desenvolvida a partir de estudos em animais que consideram peso e a característica animal; 7) a suscetibilidade genética dos envolvidos, tanto a mãe quanto o feto em questão, pois mesmo com número reduzido de estudos e a falta de comprovação específica para esta característica, 
sabe-se que existem diferenças genéticas importantes entre indivíduos brancos e afro-descendentes em relação aos efeitos teratogênicos do tabagismo por exemplo (7,19-21,24).

Atualmente estão descritos seis mecanismos de ação pelos quais os fármacos podem se comportar como agentes teratogênicos $(25,26)$ :

Antagonismo do folato. Ocorrem duas reações de redução na conversão do folato: a primeira converte o folato em sua forma bioativa, o hidrofolato (THF), transformação realizada pela di-hidrofolato redutase (DHFR); a segunda converte o THF em 5-metiltetrahidrofolato monoglutamato (5-MTHF). O 5-MTHF é a principal forma de folato circulante e é transportado para as células por três vias: por receptores associados à membrana; por um sistema mediado por transportador; e por difusão passiva. Este metabólito atua como uma coenzima essencial em reações bioquímicas por mediar a troca de unidades de carbono. As principais reações envolvidas estão relacionadas com a síntese de purina, pirimidina e a metilação de DNA. Essas reações são essenciais para o crescimento e desenvolvimento fetal e requerem incremento de folato durante a gravidez $(13,27,28)$.

Os fármacos que atuam por antagonismo do folato consistem em inibidores competitivos da enzima DHFR e incluem metotrexato, triamtereno e trimetoprim que bloqueiam a conversão de ácido fólico para THF por ligação irreversível à enzima. Estudos evidenciam que outro grupo de fármacos atue no metabolismo do folato, prejudicando tanto a absorção quanto aumentando a degradação do mesmo. Esse grupo é composto principalmente por medicamentos anticonvulsivantes, incluindo carbamazepina, ácido valproico e fenitoína $(26,29)$.

Rompimento das células da crista neural. A crista neural se origina da fusão da placa neural com o tecido ectodérmico não neural. Ela é constituída por grande quantidade de células pluripotentes e pode ser dividida em duas populações principais: da crista neural cranial e do tronco $(25,30,31)$.

Durante a neurulação, as células da crista neural migram para o embrião para dar origem a numerosas estruturas celulares, incluindo ossos, cartilagens, nervos e músculos. A crista neural cardíaca é uma subpopulação da crista neural cranial, que migra para o tecido cardíaco, e pode ainda mediar a formação de septos e outros derivados da faringe, tais como o timo, a tireoide e as glândulas paratireoides. Defeitos na formação, nas glândulas da faringe, esôfago, tireoide, defeitos ósseos e malformações cranianas cardiovasculares, são geralmente atribuídos ao rompimento da crista neural $(26,30)$. Agem por este mecanismo teratogênico todos os derivados retinoicos tais como: tretinoína, isotretinoína e o etretinato $(18,26)$.

Desreguladores endócrinos dos hormônios sexuais. Desregulador endócrino é o "agente exógeno que interfere com a síntese, secreção, transporte, ligação, ação ou eliminação de hormônio natural no corpo, responsável pela manutenção, reprodução, desenvolvimento e/ou comportamento dos organismos" (32). Mediante o amplo conceito e a influência de outros mecanismos teratogênicos subjacentes, torna-se difícil detectar e comprovar de forma experimental, em humanos, a dose e os efeitos diretos destes desreguladores, mas diversos estudos epidemiológicos e experimentais com animais apontam para associação dessas substâncias com várias anomalias ligadas, principalmente, aos fetos masculinos e na formação da genitália externa $(18,26,32,33)$. Os ftalatos presentes nos revestimentos de fármacos como omeprazol, os hormônios sintéticos, os anticoncepcionais hormonais e os fármacos destinados ao tratamento de infertilidade estão entre os principais representantes desse grupo, e aparecem relacionados a anomalias de genitália externa, hipospádia e criptorquidia $(18,26)$.

Estresse Oxidativo. Todos os organismos vivos possuem uma capacidade de manter uma perfeita harmonia entre a produção de espécies reativas de oxigênio e a sua eliminação, por remoção ou redução de danos por elas causados. Quando ocorre o desequilíbrio desse sistema, o organismo entra em um processo conhecido como estresse oxidativo $(30,33,34)$.

As espécies reativas de oxigênio circulantes promovem ligação covalente ou irreversível com macromoléculas de DNA, proteínas e lipídios, conduzindo à inativação de muitas enzimas e morte celular. Além de ser prejudicial para as macromoléculas, o estresse oxidativo pode afetar a expressão do gene, interferindo com a atividade de fatores de transcrição e sinal de transdução. Durante o período pré-natal, isto pode resultar em anomalias congênitas, retardo no crescimento e em casos graves, morte intrauterina $(26,29,34)$.

Rompimento vascular. Consiste em anomalias congênitas decorrentes de alterações vasculares ocorridas principalmente no primeiro trimestre gestacional. A etiologia proposta parte de dois mecanismos: um é a via extrínseca em que se acredita na ruptura precoce do âmnio, e o outro é a via intrínseca, a qual aparece intimamente ligada à teratogenicidade dos medicamentos, em que exposição gestacional a substâncias vasoativas, 
principalmente vasoconstritores, tem sido apontada como fator causal na formação de defeitos vasculares. Estudos sugerem que estes teratógenos estão relacionados com a diminuição do fluxo sanguíneo placentário ou fetal, afetando tanto a estrutura quanto a anatomia de vasos sanguíneos $(26,35,36)$. Os principais medicamentos representantes desse grupo são anti-hipertensivos, misoprostol, ácido acetilsalicílico, ergotamina e pseudoefedrina (29).

A afinidade de teratógenos por receptores ou enzimas específicas pode levar a inibição ou estimulação das mesmas. As principais enzimas associadas a esse efeito são: 1) perturbação do sistema renina-angiotensina fetal, produzindo hipotensão fetal, rompimento vascular e diminuição do tônus vascular renal fetal, quando da administração de inibidores da enzima conversora de angiotensina (ECA) e antagonistas dos receptores de angiotensina II (ARA); 2) as estatinas inibem a hidroximetilglutaril-coenzima A (HMG-CoA) redutase, a enzima limitante da velocidade na via que converte HMG-CoA em ácido mevalônico - a inibição desta via promovida por estatinas pode conduzir a uma ampla gama de defeitos que vão desde o crescimento embrionário até formação de estruturas; 3) a desacetilação das histonas por inibição da enzima histona desacetilase (HDACs) compromete uma grande quantidade de funções celulares, incluindo a regularidade da expressão gênica por remodelação da cromatina, a transcrição para o DNA, resultando em interrupção da proliferação celular, diferenciação e apoptose; 4) a inibição da ciclooxigenase I (COX-I) que catalisa a conversão do ácido araquidônico a prostaglandinas, interferindo no sistema renina-angiotensina, nesse grupo se encaixam todos os antiinflamatórios não esteroidais. 5) o bloqueio do receptor $N$-metil- $D$-aspartato (NMDA), causando erros na migração de elementos neuronais e gliais no cérebro em desenvolvimento; 6) o aumento ou a supressão da estimulação de receptores da 5-hidroxitriptamina ou a inibição do transporte pode causar anomalias congênitas e os fármacos envolvidos neste mecanismo são a buspirona e risperidona; 7) a inibição da anidrase carbônica, causando redução no $\mathrm{pH}$ intracelular embrionário sendo os fármacos envolvidos a acetazolamida e o topiramato $(18,25,26,28,29,33,37-39)$.

Os efeitos dos fármacos utilizados pela mãe durante gestação são bastante investigados, porém achados recentes alertam para os riscos teratogênicos do uso de medicamentos pelos pais durante o período pré-concepcional. Os mecanismos envolvidos podem ser por ações mutagênicas diretas, distúrbios na espermatogênese ou a transferência de metabólitos ativos para a mãe por meio do sêmen. Apesar de haver inconsistência de alguns achados, estudos epidemiológicos sugerem a associação entre o uso de diazepan pelo pai e o risco aumentado de mortalidade perinatal e retardo de crescimento intrauterino $(40,41)$. Ainda sobre as alterações com a espermatogênese estudos apontam o uso da finasterida como risco para anomalias congênitas (42)

Exposição aos medicamentos de risco. Devido à histórica escassez de ensaios clínicos controlados com gestantes, informações obtidas por estudos observacionais ainda são consideradas como padrão para classificação e definição de teratogenicidade, desde que o estudo tenha um excelente delineamento e confiabilidade. Outro ponto que corrobora a aceitação dos estudos observacionais é o fato de que os dados obtidos a partir de estudos com animais não podem ser, integralmente, extrapolados para a gravidez humana $(20,43)$.

Apesar de diversos estudos envolverem os mecanismos teratogênicos, a farmacocinética e a farmacodinâmica dos medicamentos na gestante, o potencial de risco dos fármacos ainda não são conhecidos por meio de estudos experimentais em humanos, exceto para aqueles já classificados como de alto risco, desde os ensaios clínicos com animais. Sendo assim, o ideal seria que não houvesse a utilização de medicamentos por gestantes, mas sabe-se que isso raramente ocorre, ou seja, as gestantes estão expostas a diferentes grupos farmacológicos que vão desde as vitaminas, agentes anti-infecciosos, analgésicos, antiasmáticos, produtos dermatológicos e até mesmo antidepressivos e ansiolíticos em alguns casos (44)

A relação risco/benefício deve sempre prevalecer na decisão do prescritor em tratar ou não uma gestante. Para uma tomada de decisão sobre uma terapia medicamentosa é sempre aconselhável consultar a literatura especializada, o que poderá ser feito diretamente às fontes de consulta ou por meio dos centros de informação em medicamentos, tanto nas bases nacionais como internacionais.

A utilização indiscriminada e as dificuldades de conciliação de prescrições para gestantes que necessitam de tratamento medicamentoso, impulsionou a Food and Drug Administration (FDA), agência reguladora de medicamentos e alimentos dos Estados Unidos, a elaborar, em 1979, um regulamento que estabelece uma categorização que descreve os riscos oferecidos pelos medicamentos a serem utilizados por mulheres durante 
o período gestacional. Estas indicações necessariamente devem ser incluídas nas bulas, além de estarem disponíveis aos profissionais de saúde, visando a orientação correta e segura quanto à prescrição e utilização dos fármacos $(19,20,45)$.

Em 1997, ciente de que esse regulamento apresentava falhas e inconsistências, o FDA lançou uma consulta pública, que resultou em uma nova proposta. Considerando que a absorção e o metabolismo dos fármacos depende de diversos fatores e que uma categoria baseada apenas em estudos com animais não referenda princípios de segurança, o Comitê de pesquisa definiu que as bulas dos fármacos deveriam conter no mínimo três grupos de informações: sumário de riscos, considerações clínicas e de dados $(7,14,20)$.

A seção de sumários de risco apresenta a probabilidade de efeitos adversos dos fármacos e pode incluir informações para diferentes riscos. As considerações clínicas deverão conter informações importantes para prescritores, auxiliando no aconselhamento adequado, informações sobre exposição acidental, ajustes de dosagem, opções de tratamento e efeitos adversos que podem surgir devido ao uso na gravidez. A seção de considerações de dados deverá incluir informações detalhadas sobre estudos e ensaios clínicos de fármacos, apresentando inicialmente dados de ensaios clínicos com humanos e posteriormente com animais. Esta nova regra de rotulagem para gravidez e lactação foi proposta em 2008 pela FDA e encontra-se ainda em processo de liberação $(20,46)$.

No Brasil, as regras de classificação de medicamentos ainda permanecem de acordo com as cinco categorias de risco descritas pelo FDA:

Categoria A: fármacos para os quais não foram constatados riscos para o feto em ensaios clínicos cientificamente desenhados e controlados;

Categoria $B$ : fármacos para os quais os estudos com animais de laboratório não demonstraram risco fetal (mas não existem estudos adequados em humanos) e cujos estudos com animais indicaram algum risco, mas que não foram comprovados em humanos em estudos devidamente controlados;

Categoria $C$ : fármacos para os quais os estudos em animais de laboratório revelaram efeitos adversos ao feto, mas não existem estudos adequados em humanos e para os quais não existem estudos disponíveis;

Categoria $D$ : fármacos para os quais a experiência de uso durante a gravidez mostrou associação com o aparecimento de más formações, mas que a relação risco/benefício pode ser avaliada;

Categoria $X$ : fármacos associados com anormalidades fetais em estudos com animais e em humanos e ou cuja relação risco/benefício contra-indica seu uso na gravidez (47).

\section{CONCLUSÃO}

A utilização de fármacos na gestação expõe a mãe e o feto a riscos, sejam eles determinados pelas necessidades terapêuticas próprias da gestação, em função de morbidades pré-existentes, bem como por intercorrências obstétricas. Neste sentido amplas revisões narrativas da literatura possuem papel fundamental, pois podem agregar um grande número de informações científicas, propiciando aos profissionais envolvidos na assistência à saúde materna e infantil adquirir e atualizar o conhecimento sobre a farmacocinética e teratogenicidade dos fármacos na gestação em um curto espaço de tempo. 


\section{REFERÊNCIAS}

1. França ISX de, Simplício D da N, Alves FP, Brito VR de S. Vaccination coverage and childmortality in Campina Grande, PB, Brazil. Rev Bras Enferm. 2009; 62(2):258-271.

2. Luhm KR, Cardoso MRA, Waldman EA. Cobertura vacinal em menores de dois anos a partir de registro informatizado de imunização em Curitiba, PR. Rev Saúde Pública. 2011; 45:90-98.

3. Koren G. Pharmacokinetics in pregnancy; clinical significance. J Popul Ther Clin Pharmacol. 2011; 18(3):e523-527.

4. Steinkellner A, Chen W, Denison SE. Adherence to oral contraception in women on category $\mathrm{X}$ medications. Am J Med. 2010; 123(10):929-934.

5. Schuler-Faccini L, Leite JCL, Sanserino MTV, Peres RM. Avaliação de teratógenos na população brasileira. Ciênc Saúde Coletiva. 2002; 7(1):65-71.

6. Anderson GD. Pregnancy-induced changes in pharmacokinetics: a mechanistic-based approach. Clin Pharmacokinet. 2005; 44(10):989-1008.

7. Feibus KB. FDA's proposed rule for pregnancy and lactation labeling: improving maternal child health through well-informed medicine use. J Med Toxicol. 2008; 4(4):284-288.

8. Hebert MF. Impact of pregnancy on pharmacokinetics of medications. J Popul Ther Clin Pharmacol. 2013; 20(3):350-357.

9. Ke AB, Rostami-Hodjegan A, Zhao P, Unadkat JD. Pharmacometrics in Pregnancy: An Unmet Need. Annu Rev Pharmacol Toxicol. 2014; 54(1):53-69.

10. Holmes LB. Human teratogens: Update 2010. Birt Defects Res - A Clin Mol Teratol; 2011; 91(1):1-7.

11. Goodman LS, Brunton LL, Chabner B, Knollmann BC, editors. Goodman \& Gilman's pharmacological basis of therapeutics. 12th ed. New York: McGraw-Hill; 2011. 2084 p.

12. Costantine MM. Physiologic and pharmacokinetic changes in pregnancy. Front Pharmacol. 2014; 5: 65.

13. Katzung BG, Masters SB, Trevor AJ. Basic and clinical pharmacology. 12th ed. Porto Alegre: AMGH; 2014. $1242 \mathrm{p}$.

14. Knoppert D. Safety and efficacy of drugs in pregnancy. J Popul Ther Clin Pharmacol J Thérapeutique Popul Pharamcologie Clin. 2011; 18(3):e506-512.

15. Dal Pizzol TS. Riscos e benefícios para o feto e recém-nascido de medicamentos utilizados na gestação: misoprostol e antianêmicos. [Porto Alegre]: Programa de Pós-graduação em Epidemiologia. Faculdade de Medicina, Universidade Federal do Rio Grande do Sul; 2006.

16. Emanuel M, Rawlins M, Duff G, Breckenridge A. Thalidomide and its sequelae. The Lancet. 2012; 380(9844):781-783.

17. McBride WG. Thalidomide and congenital abnormalities. The Lancet. 1961; 278(7216): 1358.

18. Običan S, Scialli AR. Teratogenic exposures. In: American Journal of Medical Genetics Part C: Seminars in Medical Genetics. Wiley Subscription Service, Inc., Wiley Company, 2011: 150-169.
19. Rasmussen SA. Human teratogens update 2011: Can we ensure safety during pregnancy? Birt Defects Res - Clin Mol Teratol. 2012; 94(3):123-128.

20. Holmes LB. Human teratogens: Update 2010. Birt Defects Res - Clin Mol Teratol. 2011; 91(1):1-7.

21. Morgan M, De Jong-Van Den Berg LT, Jordan S. Drug safety in pregnancy-monitoring congenital anomalies. J Nurs Manag. 2011; 19(3):305-310.

22. Shahin I, Einarson A. Knowledge transfer and translation: Examining how teratogen information is disseminated. Birt Defects Res - Clin Mol Teratol. 2011; 91(11):956-961.

23. EUROCAT. A review of environmental risk factors for congenital anomalies: special report. Newtownabbey: EUROCAT; 2004.

24. Adam MP, Polifka JE, Friedman JM. Evolving knowledge of the teratogenicity of medications in human pregnancy. Am J Med Genet C Semin Med Genet. 2011; 15;157(3):175-182.

25. van Gelder MMHJ, de Jong-van den Berg LTW, Roeleveld N. Drugs associated with teratogenic mechanisms. Part II: a literature review of the evidence on human risks. Hum Reprod. 2014; 29(1):168-183.

26. van Gelder MMHJ, van Rooij IALM, Miller RK, Zielhuis GA, de Jong-van den Berg LTW, Roeleveld N. Teratogenic mechanisms of medical drugs. Hum Reprod Update. 2010; 16(4):378-394.

27. Carmichael SL, Yang W, Shaw GM. Periconceptional nutrient intakes and risks of neural tube defects in California. Birt Defects Res - Clin Mol Teratol. 2010; 88(8):670-678

28. Silva P. Farmacologia. Rio de Janeiro: Guanabara Koogan; 2010.

29. Van Gelder MMHJ, Bos JHJ, Roeleveld N, de Jong-van den Berg LTW. Drugs associated with teratogenic mechanisms. Part I: dispensing rates among pregnant women in the Netherlands, 1998-2009. Hum Reprod. 2014; 29(1):161-167.

30. Moore KL, Persaud TVN, Torchia MG. Embriologia Básica. 8nd. ed. São Paulo. 2013.

31. Schmidt AG, Prosdócimi FC. Manual de neuroanatomia humana guia prático. 1nd. ed. Grupo Gen - Editora Roca Ltda.; Curitiba. 2014.

32. Bila DM, Dezotti M. Desreguladores endócrinos no meio ambiente: efeitos e consequências. Quím Nova. 2007; 30(3):651

33. Marques ECM, Martins AC. Anatomia e fisiologia humana. 2nd. ed. Martinari. São Paulo. 2015.

34. Barbosa KBF, Costa NMB, Alfenas R de CG, De Paula SO, Minim VPR, Bressan J. Oxidative stress: concept, implications and modulating factors. Rev Nutr. 2010; 23(4):629-643

35. Nogueira FCS, da Cruz RB, Machado LP, Ramos BLF, Júnior JLM, de Almeida Pinto RZ. Síndrome da banda amniótica: relato de caso. Rev Bras Ortop. 2011; 46(4):56-62. 
36. Saldarriaga-Gil W, Ayala-Zapata S, Ramírez-Cheyne JA, Isaza C. Pentalogía de Cantrell y bandas amnióticas: reporte de un caso y revisión de la literatura. Rev Colomb Obstet Ginecol. 2014; 65(3):243-249.

37. van Gelder M, Van Bennekom C, Louik C, Werler M, Roeleveld N, Mitchell A. Maternal hypertensive disorders, antihypertensive medication use, and the risk of birth defects: a case-control study. BJOG Int J Obstet Gynaecol. 2015; 122(7):1002-1009.

38. Sato R, Ikuma M, Takagi K, Yamagishi Y, Asano J, Matsunaga $\mathrm{Y}$, et al. Exposure of Drugs for Hypertension, Diabetes, and Autoimmune Disease During Pregnancy and Perinatal Outcomes: An Investigation of the Regulator in Japan. Medicine (Baltimore). 2015; 94(1):e386.

39. Bellantuono C, Tofani S, Di Sciascio G, Santone G. Benzodiazepine exposure in pregnancy and risk of major malformations: a critical overview. Gen Hosp Psychiatry. 2013; 35(1):3-8.

40. Engeland A, Bramness JG, Daltveit AK, Rønning M, Skurtveit S, Furu K. Prescription drug use among fathers and mothers before and during pregnancy. A population-based cohort study of 106000 pregnancies in Norway 2004-2006. Br J Clin Pharmacol. 2008; 65(5):653-660.

41. Engeland A, Bjørge T, Daltveit AK, Skurtveit S, Vangen $\mathrm{S}$, Vollset SE, et al. Effects of preconceptional paternal drug exposure on birth outcomes: cohort study of 340 000 pregnancies using Norwegian population-based databases: Effects of paternal drug exposure on birth outcomes. Br J Clin Pharmacol. 2013; 75(4):1134-1141.

42. Hirshburg JM, Kelsey PA, Therrien CA, Gavino AC, Reichenberg JS. Adverse Effects and Safety of 5-alpha Reductase Inhibitors (Finasteride, Dutasteride): A Systematic Review. J Clin Aesthetic Dermatol. 2016; 9(7):56-62.

43. Rodrigues AVP, Terrengui LCS. Uso de medicamentos durante a gravidez. Rev Enferm UNISA. 2006; 7:9-14.

44. Rocha RS, Bezerra SC, Lima JWO, Costa FS. Consumo de medicamentos, álcool e fumo na gestação e avaliação dos riscos teratogênicos. Rev Gaúcha Enferm. 2013; 34(2):37-45.

45. Ribeiro NKR, Leite LLB, da Silva Pontes ZBV. Estudo farmacoepidemiologico: o uso de medicamentos por gestantes. Rev Eletron Farmácia. 2013; 10(1):11.

46. Ramoz LL, Patel-Shori NM. Recent changes in pregnancy and lactation labeling: retirement of risk categories. Pharmacother J Hum Pharmacol Drug Ther. 2014; 34(4):389-395.

47. FDA. Food and Drugs Administration. Prescription drug advertising: content and format for labeling of human prescription drugs. Rules and regulations. 1979; 26 (44):37451. 